Commun. Korean Math. Soc. 27 (2012), No. 2, pp. 377-384

http://dx.doi.org/10.4134/CKMS.2012.27.2.377

\title{
THE $k$-QUOTIENT IMAGES OF METRIC SPACES
}

\author{
Shou Lin And Chunyan Zheng
}

\begin{abstract}
In this paper some properties of sequentially closed sets and $k$-closed sets in a topological space are discussed, it is shown that a space is a $k$-quotient image of a metric space if and only if its each sequentially closed set is $k$-closed, and some related examples about connectedness are obtained.
\end{abstract}

\section{Introduction}

It is well known that a topological space is a $k$-space if and only if it is a quotient image of a paracompact locally compact space [5]. Shou Lin [10] raised the following question: Are connected $k$-spaces the quotient images of connected locally compact spaces? Chunyan Zheng [13] defined $k$-connected spaces, analyzed the relationship between $k$-connectedness and connected paracompact locally compact spaces by mappings, and proved that a Hausdorff space is a $k$-connected space with a point-finite $k$-system if and only if it is a quotient compact image of a connected paracompact locally compact space, which gave a partial answer to the question above. A related topological space is a sequentially connected space [6]. Every sequentially connected space is $k$-connected, and every $k$-connected space in which each sequentially closed set is $k$-closed is sequentially connected, which inspires our interest in the property of "each sequentially closed set is $k$-closed in a space". On the other hand, in order to extend the compact-covering mappings defined by A. Arhangel'skiǐ, J. R. Boone [1] defined $k$-quotient mappings in 1974, and established some results about the invariance of topological structures, but there is still an important question how to characterize precisely the $k$-quotient images of metric spaces. The main result of this paper is that the $k$-quotient images of metric spaces can be characterized as the spaces in which each sequentially closed set is $k$ closed. This enhances our interest in this property. Through studying some

Received November 9, 2010.

2010 Mathematics Subject Classification. 54B15, 54C10, 54D05, 54D50, 54D55.

Key words and phrases. sequentially closed sets, $k$-closed sets, $k$-quotient mappings, sequentially quotient mappings, connectedness.

The project is supported by the NNSF (No. 10971185) and the NSF of Fujian Province (No. 2009J01013) of China. This work has been done during the second author of this paper stay at Zhangzhou Normal University. 
of its basic properties, our knowledge on sequential spaces, quotient mappings and connectedness are improved.

In this paper all spaces are topological spaces, and they are not required to satisfy any axioms of separations. All mappings are continuous and onto. Readers may refer to [5] for unstated notations and terminologies.

\section{Sequentially closed sets}

Definition 2.1. Let $X$ be a space. A set $H \subset X$ is called $k$-closed [1] in $X$ if $H \cap K$ is closed in $K$ for each compact subset $K$ of $X$. A space is a $k$-space if its each $k$-closed set is closed.

A set $H \subset X$ is called sequentially closed [7] in $X$ if a sequence $S$ converges to $x \notin H$ in $X$, then $S$ is eventually in $X \backslash H$. A space is sequential if its each sequentially closed set is closed.

First, we discuss some characterizations of sequentially closed sets in a space.

Lemma 2.2. The following conditions are considered for a space $X$ and $P \subset$ $X$ :

(1) $P$ is sequentially closed in $X$;

(2) Each limit of a sequence in $P$ which converges in $X$ is in $P$;

(3) If $S$ is a convergent sequence in $X$ containing its a limit, then $S \cap P$ is closed in $S$.

Then $(3) \Rightarrow(2) \Leftrightarrow(1)$; and $(1) \Rightarrow(3)$ if $X$ is Hausdorff.

Proof. (3) $\Rightarrow(2)$ Suppose $P$ satisfies (3). If a sequence $\left\{x_{n}\right\} \subset P$ converges to $x$ in $X$, put $S=\{x\} \cup\left\{x_{n}: n \in \mathbb{N}\right\}$, then $x_{n} \in S \cap P$ and $S \cap P$ is closed in $S$, so $x \in S \cap P \subset P$.

$(2) \Rightarrow(1)$ Assume $P$ satisfies (2). If a sequence $\left\{y_{n}\right\}$ converges to $y \notin P$ in $X$, there are only finitely many $y_{n} \in P$ by (2), i.e., there exists $k \in \mathbb{N}$ such that $x_{n} \in X \backslash P$ whenever $n>k$. It follows that $P$ is sequentially closed in $X$.

$(1) \Rightarrow(2)$ Let $P$ be sequentially closed in $X$. If a sequence $\left\{x_{n}\right\} \subset P$ converges to $x$ in $X$, then $x \in P$ because $\left\{x_{n}\right\}$ is not eventually in $X \backslash P$.

Furthermore, if $X$ is Hausdorff, then $(1) \Rightarrow(3)$ by [9, Lemma 3.1.1].

Corollary 2.3. Each $k$-closed set is sequentially closed in a space. Then every sequential space is a $k$-space [5].

Proof. Assume $H$ is $k$-closed in a space $X$. If $S$ is a convergent sequence in $X$ containing its a limit, then $S$ is compact in $X$, thus $S \cap H$ is closed in $S$, i.e., $H$ satisfies the condition (3) of Lemma 2.2 , hence $H$ is sequentially closed in $X$.

Example 2.4. There exist a compact $T_{1}$-space $X$, a sequentially closed subset $P$ and a convergent sequence $C$ containing its a limit in $X$ such that $C \cap P$ is not closed in $C$. 
Proof. Let $\beta \mathbb{N}$ be the Stone-Čech compactification of the positive integer set $\mathbb{N}$. Take $p \in \beta \mathbb{N} \backslash \mathbb{N}$ and $q \notin \beta \mathbb{N}$. Put $X=\mathbb{N} \cup\{p, q\}$ with a topology as follows: $\mathbb{N} \cup\{p\}$ endowed with the subspace topology of $\beta \mathbb{N}$ is open in $X$, the neighborhoods of $q$ in $X$ are finite complement subsets of $X$. Then $X$ is a compact $T_{1}$-space. Since $p$ is an accumulation point of $\mathbb{N}$, every neighborhood of $p$ in $X$ contains infinitely many points of $\mathbb{N}$, thus $p, q$ can not be separated by disjoint neighborhoods in $X$. Therefore, $X$ is not Hausdorff.

According to [5, Corollary 3.6.15], there are no nontrivial convergent sequences in $\mathbb{N} \cup\{p\}$. Put $P=\mathbb{N} \cup\{q\}$. If a sequence $S \subset P$ converges to $x$ in $X$, then $x \neq p$, so $x \in P$, thus $P$ is sequentially closed in $X$ by Lemma 2.2 . Take $C=X$, then $C$ is a convergent sequence containing its a limit $q$, and $p$ is an accumulation point of $P$, thus $P=C \cap P$ is not closed in $C$.

\section{3. $k q$-spaces}

We introduce the following concept for the sake of conciseness.

Definition 3.1. A space $X$ is called a $k q$-space if each sequentially closed subset of $X$ is $k$-closed.

Lemma 3.2. $X$ is a sequential space if and only if $X$ is a $k$-, and $k q$-space.

Neither sequential spaces nor $k$-spaces are hereditary [5]. The hereditary property of $k q$-spaces is fine.

Theorem 3.3. Let $X$ be a kq-space. If each compact subset of $X$ is sequentially closed, then each subspace of $X$ is a kq-space. Hence, the property with Hausdorff $k q$-spaces is hereditary.

Proof. Let $Y$ be a subspace of $X$. If $H$ is sequentially closed in $Y$, and $H \cap K$ is not closed in $K$ for some compact subset $K \subset Y$, then $H \cap K$ is not $k$-closed in $X$, thus $H \cap K$ is not sequentially closed in $X$ because $X$ is a $k q$-space. There is a sequence $\left\{x_{n}\right\} \subset H \cap K$ converging to $x \notin H \cap K$, then $x \in K$ by Lemma 2.2 , thus $x \notin H$ and $\left\{x_{n}\right\}$ converges to $x$ in $Y$, a contradiction. Hence each sequentially closed subset is $k$-closed in $Y$, i.e., $Y$ is a $k q$-space.

Sequential spaces and quotient mappings are closely related [7], also $k q$ spaces and $k$-quotient mappings are closely related.

Definition 3.4. Let $f: X \rightarrow Y$ be a mapping.

(1) $f$ is a $k$-quotient mapping [1], if $H \subset Y$ and $f^{-1}(H)$ is $k$-closed in $X$, then $H$ is $k$-closed in $Y$.

(2) $f$ is a sequentially quotient mapping [2], if $H \subset Y$ and $f^{-1}(H)$ is sequentially closed in $X$, then $H$ is sequentially closed in $Y$.

It is easy to show that for a mapping $f: X \rightarrow Y$, if $H$ is $k$-closed (resp. sequentially closed) in $Y$, then $f^{-1}(H)$ is $k$-closed (resp. sequentially closed) in $X$. J. R. Boone [1] has given examples to show that the concepts of sequentially 
quotient mappings and $k$-quotient mappings are generally independent. What conditions will make them have some nice relationship? $k q$-spaces are a way.

Theorem 3.5. Let $f: X \rightarrow Y$ be a mapping, where $X$ is a kq-space. Then $f$ is a $k$-quotient mapping if and only if $Y$ is a kq-space and $f$ is sequentially quotient.

Proof. Let $f$ be a $k$-quotient mapping. If $H$ is sequentially closed in $Y$, then $f^{-1}(H)$ is sequentially closed in $X$. Because $X$ is a $k q$-space, $f^{-1}(H)$ is $k$ closed in $X, H$ is $k$-closed in $Y$ for $f$ is $k$-quotient. Hence $Y$ is a $k q$-space. On the other hand, if $G \subset Y$ and $f^{-1}(G)$ is sequentially closed in $X$, then $f^{-1}(G)$ is $k$-closed in $X$, thus $G$ is $k$-closed in $Y$, hence $G$ is sequentially closed in $Y$. It follows that $f$ is sequentially quotient.

Conversely, let $f$ be a sequentially quotient mapping and $Y$ a $k q$-space. If $H \subset Y$ and $f^{-1}(H)$ is $k$-closed in $X$, then $f^{-1}(H)$ is sequentially closed in $X$, thus $H$ is sequentially closed in $Y$, so $H$ is $k$-closed in $Y$. Hence $f$ is $k$-quotient.

It is known that a sequential space can be characterized as a quotient image of a metric space [7]. $k q$-spaces have a similar characterization. First, let us to check following lemma, which indicates the generality of sequentially quotient mappings.

Lemma 3.6. Every space is a sequentially quotient image of a locally compact metric space.

Proof. For any space $X$, denote the family of all convergent sequences in $X$ containing its a limit by $\left\{S_{\alpha}: \alpha \in A\right\}$. For every $\alpha \in A$, set $S_{\alpha}=\left\{x_{\alpha}\right\} \cup\left\{x_{\alpha, n}\right.$ : $n \in \mathbb{N}\}$, where $x_{\alpha, n} \rightarrow x_{\alpha}$. Denote $S_{\alpha}$ endowed with the following new topology by $S_{\alpha}^{\prime}$ : the neighborhoods of the point $x_{\alpha}$ in $S_{\alpha}^{\prime}$ are the finite complement subsets of $S_{\alpha}$, other points are isolated. Then $S_{\alpha}^{\prime}$ is a compact metric space, the topology on $S_{\alpha}^{\prime}$ is finer than the subspace topology on $S_{\alpha}$ of $X$. Let $M$ be the disjoint topological sum of the family $\left\{S_{\alpha}^{\prime}: \alpha \in A\right\}[3]$, and define a function $f: M \rightarrow X$ by $h_{\alpha}=\left.f\right|_{S_{\alpha}^{\prime}}: S_{\alpha}^{\prime} \rightarrow S_{\alpha}$ is homeomorphic for each $\alpha \in A$. Then $M$ is a locally compact metric space, $f$ is continuous and surjective. We will check that $f$ is sequentially quotient. Let $H \subset X$ and $f^{-1}(H)$ be a sequentially closed subset of $M$. If a sequence $S_{\alpha}$ converges to $x_{\alpha} \notin H$ in $X$, then $h_{\alpha}^{-1}\left(x_{\alpha}\right) \notin f^{-1}(H)$ in $M$. Since $h_{\alpha}^{-1}\left(S_{\alpha}\right)$ converges to $h_{\alpha}^{-1}\left(x_{\alpha}\right)$ in $M$, $h_{\alpha}^{-1}\left(S_{\alpha}\right)$ is eventually in $M \backslash f^{-1}(H)$, then $S_{\alpha}$ is eventually in $X \backslash H$ in $X$, thus $H$ is sequentially closed in $X$. Hence $f$ is sequentially quotient.

Theorem 3.7. The following are equivalent for a space $X$ :

(1) $X$ is a kq-space;

(2) Every sequentially quotient mapping onto $X$ is $k$-quotient;

(3) $X$ is a k-quotient image of a locally compact metric space;

(4) $X$ is a k-quotient image of a metric space. 
Proof. (1) $\Rightarrow(2)$ Let $X$ be a $k q$-space and $f: Z \rightarrow X$ a sequentially quotient mapping. If $H \subset X$ and $f^{-1}(H)$ is $k$-closed in $Z$, then $f^{-1}(H)$ is sequentially closed in $Z$. Because $f$ is sequentially quotient, $H$ is sequentially closed in $X$. Since $X$ is a $k q$-space, $H$ is $k$-closed in $X$. Hence $f$ is $k$-quotient.

$(2) \Rightarrow(3)$ Assume every sequentially quotient mapping onto $X$ is $k$-quotient. By Lemma 3.6, there exist a locally compact metric space $M$ and a sequentially quotient mapping $f: M \rightarrow X$, so $f$ is $k$-quotient.

$(3) \Rightarrow(4)$ is obvious. (4) $\Rightarrow$ (1) by Theorem 3.5.

Just as $(1) \Leftrightarrow(4)$ in Theorem 3.7 , the spaces in which "every sequentially closed set is $k$-closed" are called the $k q$-spaces in Definition 3.1. The theorem above also indicates that all of spaces is not a $k$-quotient image of a metric space.

A space $X$ has a point-G $G_{\delta}$ property if each singleton of $X$ is a $G_{\delta}$-set.

Theorem 3.8. A Hausdorff space with a point-G $G_{\delta}$ property is a $k q$-space.

Proof. Suppose $X$ is a Hausdorff space with a point- $G_{\delta}$ property and $H$ is sequentially closed in $X$. For any compact subset $K$ of $X$, if $x \in \operatorname{cl}_{K}(K \cap H)$, there exists a sequence $\left\{x_{n}\right\}$ in $K \cap H$ which converges to $x$ because a compact Hausdorff space with a point- $G_{\delta}$ property is a first countable space (see [5, 3.1.F(b)]). Since $K$ is closed and $H$ is sequentially closed in $X, x \in K \cap H$, i.e., $K \cap H$ is closed in $K$, then $H$ is $k$-closed in $X$. It implies that $X$ is a $k q$-space.

Remark 3.9. The Hausdorff property in Theorem 3.8 is essential. The space $X$ in Example 2.4 is a $T_{1}$-space with a point- $G_{\delta}$ property because $X$ is a countable set. Since the set $P$ in Example 2.4 is a sequentially closed and non- $k$-closed subset of $X, X$ is not a $k q$-space.

A space $X$ is of countable tightness if $x \in \bar{A} \subset X$, there exists a countable set $C \subset A$ such that $x \in \bar{C}$. It is known that a sequential space is of countable tightness (see $[5,1.7 .13(\mathrm{c})]$ ). What is the relationship between $k q$ spaces and spaces of countable tightness? It is still an open problem posed by Moore-Mrówka [11] "Is every compact Hausdorff space of countable tightness sequential?" So it is difficult to seek a space of countable tightness which is not a $k q$-space.

Example 3.10. The Fortissimo space [12]: a $k q$-space without countable tightness.

Proof. For any uncountable set $X$, take $p$ as a particular point of $X . X$ is called a Fortissimo space if $X$ is endowed with the following topology: $F \subset X$ is closed in $X$ if and only if $p \in F$ or $F$ is countable. Then $X$ is a regular Lindelöf space. Since $p \in \overline{X \backslash\{p\}}$ and $p \notin F=\bar{F}$ for any countable $F \subset X \backslash\{p\}$, then $X$ is of no countable tightness.

If $K$ is an infinite compact subset of $X$, we can assume that $p \in K$, and choose an infinite subset $\left\{x_{n}: n \in \mathbb{N}\right\}$ of $K \backslash\{p\}$, then the open covering 
$\left\{X \backslash\left\{x_{n}: n \in \mathbb{N}\right\}\right\} \cup\left\{\left\{x_{n}\right\}: n \in \mathbb{N}\right\}$ of $K$ has no finite subcovering, a contradiction. So all compact subsets of $X$ are finite, then each subset of $X$ is $k$-closed, thus $X$ is a $k q$-space.

It is easy to check that $X$ has no point- $G_{\delta}$ property. Hence the converse proposition of Theorem 3.8 in a Hausdorff space does not hold.

\section{4. $k$-connectedness}

Á. Császár [4] introduced the concept of $\gamma$-connected sets. The theory of $\gamma$-connected sets is adapt to many connected-like spaces defined by special subsets. Two kinds of connectedness which are relate to sequentially closed sets and $k$-closed sets are introduced as follows.

Definition 4.1. Let $X$ be a space.

(1) $X$ is sequentially connected [6], if $X$ can not be expressed as the union of two nonempty disjoint sequentially closed subsets of $X$.

(2) $X$ is $k$-connected [13], if $X$ can not be expressed as the union of two nonempty disjoint $k$-closed subsets of $X$.

Q. Huang, S. Lin [8], and C. Zheng [13] discussed some properties of connected spaces, sequentially connected spaces and $k$-connected spaces. Some elemental relationships are obtained by Definition 4.1 and Corollary 2.3. For examples, every sequentially connected space is $k$-connected; every $k$-connected space is connected. The following Examples 4.2 and 4.3 will prove that the converse are false. However, a connected $k$-space is $k$-connected; a $k$-connected $k q$-space is sequentially connected.

Example 4.2. A Tychonoff connected $k q$-space is not $k$-connected.

Proof. Let $\beta \mathbb{R}$ be the Stone-Čech compactification of real line $\mathbb{R}$. Put $X=$ $\mathbb{R} \cup\{p\}$, where $p \in \beta \mathbb{R}-\mathbb{R}$, and endow $X$ with the subspace topology of $\beta \mathbb{R}$. Then $X$ is a Tychonoff space. Because $\mathbb{R}$ is a connected and dense subset in $X$, $X$ is connected. Now, we show that $\mathbb{R}$ is $k$-closed in $X$. If $K$ is a compact subset of $X$, then $|K| \leqslant c$, and $K \cap \mathbb{R}$ is a bounded set in $\mathbb{R}$. Suppose not, we can assume that $\mathbb{N} \subset K \cap \mathbb{R}$. Because $\mathbb{N}$ is a closed subset of $\mathbb{R}, \beta \mathbb{N}$ is homeomorphic to $\operatorname{cl}_{\beta \mathbb{R}} \mathbb{N} \subset K$ by [5, Corollary 3.6.8], then $|K| \geqslant 2^{c}$ by [5, Theorem 3.6.14], a contradiction. Therefore, there are $a, b \in \mathbb{R}$ such that $K \subset[a, b] \cup\{p\}$, then $K \cap \mathbb{R}=K \cap[a, b]$ is compact, thus $K \cap \mathbb{R}$ is closed in $\mathbb{R}$. Hence $\mathbb{R}$ is $k$-closed in $X$. Since $\{p\}$ is $k$-closed in $X$ and $X=\mathbb{R} \cup\{p\}, X$ is not $k$-connected.

Let $H$ be sequentially closed and $K$ compact in $X$. Put $L=H \cap K$. Then $L$ is sequentially closed in $X$. If $p \notin L$, then $L$ is sequentially closed in $\mathbb{R}$, thus $L$ is closed in $\mathbb{R}$ and $L \subset K \cap \mathbb{R}$, so $L$ is compact in $X$. If $p \in L$, then $p \notin L \cap \mathbb{R}$ and $L \cap \mathbb{R}$ is sequentially closed in $X$ because $\mathbb{R}$ is $k$-closed in $X$, thus $L \cap \mathbb{R}$ is compact in $X$, hence $L=(L \cap \mathbb{R}) \cup\{p\}$ is compact in $X$. Therefore, $L$ is closed in $K$. This implies that $X$ is a $k q$-space. 
In the example above, $\mathbb{R}$ is $k$-closed in $X$, then $\mathbb{R}$ is sequentially closed in $X$ by Corollary 2.3. According to Lemma 2.2, if a sequence $S$ in $\mathbb{R}$ converges to a point $x$ in $\beta \mathbb{R}$, then $x \in \mathbb{R}$. This shows that $\mathbb{R}$ is sequentially closed in $\beta \mathbb{R}$.

Example 4.3. A Hausdorff compact $k$-connected space is not sequentially connected.

Proof. Take the Stone-Čech compactification $\beta \mathbb{R}$ as the example. Because $\mathbb{R}$ is a connected and dense subset in $\beta \mathbb{R}, \beta \mathbb{R}$ is connected. Since $\beta \mathbb{R}$ is compact, $\beta \mathbb{R}$ is a $k$-space, then $\beta \mathbb{R}$ is $k$-connected. We will show that $\beta \mathbb{R}$ is not sequentially connected. Obviously, $\mathbb{R}$ is open in $\beta \mathbb{R}$, then $\beta \mathbb{R} \backslash \mathbb{R}$ is closed, thus $\beta \mathbb{R} \backslash \mathbb{R}$ is sequentially closed in $\beta \mathbb{R}$. This implies that $\beta \mathbb{R}$ can be expressed as the union of two disjoint sequentially closed subsets $\beta \mathbb{R} \backslash \mathbb{R}$ and $\mathbb{R}$. Hence, $\beta \mathbb{R}$ is not sequentially connected.

In the example above, $\mathbb{R}$ is sequentially closed in $\beta \mathbb{R}$, but $\mathbb{R}$ is not $k$-closed in $\beta \mathbb{R}$.

\section{References}

[1] J. R. Boone, On k-quotient mappings, Pacific J. Math. 51 (1974), no. 2, 369-377.

[2] J. R. Boone and F. Siwiec, Sequentially quotient mappings, Czechoslovak Math. J. 26(101) (1976), no. 2, 174-182.

[3] C. R. Borges, A note on dominated spaces, Acta Math. Hungar. 58 (1991), no. 1-2, $13-16$.

[4] Á. Császár, $\gamma$-connected sets, Acta Math. Hungar. 101 (2003), no. 4, 273-279.

[5] R. Engelking, General Topology, Heldermann Verlag, Berlin, 1989.

[6] A. Fedeli and A. Le Donne, On good connected preimages, Topology Appl. 125 (2002), no. 3, 489-496.

[7] S. P. Franklin, Spaces in which sequences suffice, Fund. Math. 57 (1965), no. 1, 107-115.

[8] Q. Huang and S. Lin, Notes on sequentially connected spaces, Acta Math. Hungar. 110 (2006), no. 1-2, 159-164.

[9] S. Lin, Topologies of Metric Spaces and Function Spaces, Chinese Science Press, Beijing, 2004.

[10] _ Some problems on generalized metrizable spaces, In: E. Pearl ed., Open Problems in Topology II, Elsevier Science B. V., Amsterdam, 2007, 731-736.

[11] P. J. Nyikos, Classic problems, In: E. Pearl ed., Problems from Topology Proceedings, Topology Atlas, Toronta, 2003, 69-89.

[12] L. A. Steen and Jr J. A. Seebach, Counterexamples in Topology, Second Edition, Springer-Verlag, New York, 1978.

[13] C. Zheng, On k-connected spaces, Far East J. Math. Sci. 25 (2007), no. 1, 37-48.

SHOU LiN

Department of Mathematics

ZhANGZHOU NORMAL UNIVERSITY

Zhangzhou 363000, P. R. China

AND

INSTITUTE OF MATHEMATICS

Ningde Teachers' College

Ningde 352100, P. R. ChinA

E-mail address: shoulin60@163.com 
Chunyan Zheng

Institute of Mathematics

Ningde Teachers' College

Ningde 352100, P. R. China

E-mail address: zhengchunyan211@163.com 